\title{
Ethical considerations in international HIV vaccine trials: summary of a consultative process conducted by the Joint United Nations Programme on HIVIAIDS (UNAIDS)
}

Dale Guenter, Jose Esparza and Ruth Macklin, McMaster University, Hamilton, Canada, foint United Nations Programme on HIVIAIDS (UNAIDS), Geneva, Switzerland, Albert Einstein College of Medicine, New York, USA respectively and UNAIDS Ethical Review Committee, Geneva, Switzerland

\begin{abstract}
Research that is initiated, designed or funded by sponsor agencies based in countries with relatively high social and economic development, and conducted in countries that are relatively less developed, gives rise to many important ethical challenges. Although clinical trials of HIV vaccines began ten years ago in the US and Europe, an increasing number of trials are now being conducted or planned in other countries, including several that are considered "developing” countries. Safeguarding the rights and welfare of individuals participating as research subjects in developing countries is a priority. In September, 1997, the foint United Nations Programme on HIVIAIDS (UNAIDS) embarked on a process of international consultation; its purpose was further to define the important ethical issues and to formulate guidance that might facilitate the ethical design and conduct of HIV vaccine trials in international contexts. This paper summarises the major outcomes of the UNAIDS consultative process.

(Fournal of Medical Ethics 2000;26:37-43)
\end{abstract}

Keywords: HIV vaccine; clinical trials; research ethics; international research

\section{Introduction}

As we approach the beginning of the third decade of the HIV epidemic, it is clear that developing an effective HIV vaccine presents a formidable challenge. As of the end of 1999, while therapeutic pharmaceutical research has made major advances, only two phase III efficacy trials of closely related candidate HIV vaccines have been initiated, in the US and Thailand. Many candidate HIV vaccines have proceeded to the point of testing for safety and immunogenicity in human subjects (phase I and II clinical trials), but multiple factors have slowed progression to the final step of randomised, controlled, large-scale, phase III efficacy trials. The challenges have been scientific, logistical, political and economic.

Planning the appropriate design and location for HIV vaccine trials gives rise to ethical issues that require special attention. The HIV epidemic is characterised by distinctive biological and social factors that must be considered in the harm/ benefit analysis for individuals participating in HIV vaccine research. First, the global prevalence of disease and death related to HIV is increasing at a rate unmatched by any other agent. Although AIDS was unknown in 1980, HIV is today the most important infectious disease in the world. AIDS is now the major cause of death in Africa, and fourth worldwide. The burden of disease has been greatest in the poorest countries; more than $95 \%$ of all HIV infections occur in developing countries, and Africa is home to two out of every three people living with HIV or AIDS. Despite intense national and international efforts to control the HIV pandemic, more than 16,000 HIV infections occur every day. Treatments currently available are inadequate since they do not lead to cure, but at best slow the progression of disease. The most effective treatment antiretroviral medication - is complicated to administer, requires close medical monitoring, can cause significant adverse effects and is extremely costly. These logistical and economic barriers render treatment inaccessible for many populations, creating a sense of urgency to develop a safe, effective and globally accessible HIV preventive vaccine to complement other strategies.

$\mathrm{HIV}$ is also unique in that development of an effective vaccine will likely require that clinical trials be conducted among numerous different 
populations, including some with a relatively low level of social and economic development. This is true for several reasons. First, the large majority of HIV infections occur in developing countries, and phase III efficacy trials will need to be conducted in populations with a high incidence of new HIV infections in order to produce valid and timely results. High incidence populations in industrialised countries are already participating in current trials, but the incidence in many developing countries is much higher and affects larger populations. Second, the genetic and antigenic variability of HIV may require that candidate vaccines be tested in different areas of the world where different HIV strains are prevalent. It is possible, though not yet known, that a vaccine preventing infection with one HIV subtype may not prevent infection with another HIV subtype. Finally, it may be necessary to evaluate the efficacy of candidate vaccines in a variety of populations where the predominant route of transmission of the virus differs, and where different cofactors could influence vaccine protection.

The unique social consequences of HIV infection must also be considered in the ethical analysis of vaccine trials. People infected with HIV have experienced stigma, marginalisation, and discrimination in many forms. Volunteers participating in HIV vaccine trials may be falsely identified as HIV-positive simply through their association with a trial, or by developing falsely positive HIV antibody tests as a result of receiving candidate vaccines. Thus, the risk of social and psychological harm for human subjects participating in vaccine research is substantial.

While previous ethical statements have articulated the principles of ethical research, ${ }^{1-4}$ and some have provided guidance on the application of these principles to international research settings, ${ }^{23}$ several of the issues emerging from HIV vaccine trials have illuminated important gaps. The Joint United Nations Programme on HIV/AIDS (UNAIDS) has identified these areas of ethical uncertainty as a priority for accelerated discussion and formulation of relevant guidance.

\section{The consultation process}

The process began with a consultation in Geneva in September 1997. Representatives from developed and developing countries began to identify the major ethical challenges that needed to be confronted in relation to international HIV vaccine research. The result of this meeting was a plan to organise regional workshops in order to seek views from communities and interested parties around the world. Three countries representing different geographical regions were invited to host these workshops in April and May, 1998. Brazil, Thailand and Uganda were chosen because of their previous involvement in HIV vaccine trials, their familiarity with the relevant scientific and social issues, and their imminent need to develop greater familiarity with the ethical implications of vaccine research. Biomedical and social scientists, community members, nongovernment organisations, activists, persons living with HIV/AIDS, ethicists, lawyers and government representatives participated in the workshops. An average of thirty participants representing the host country and other countries in the region attended each of the three workshops, which lasted three days.

\section{Several objectives}

The workshops had several objectives. The first was to familiarise participants with important scientific details related to HIV vaccines and the design of clinical trials. The second was to discuss relevant social, political and economic conditions, and economic conditions in the local context that would have ethical implications for proposed vaccine research. Finally, the intent was to establish a continuing discourse on HIV vaccine ethics both locally and in the international community.

A hypothetical case-scenario was used to facilitate workshop discussions. This case was framed as an HIV preventive vaccine trial proposal that involved a sponsor company or agency from a developed country, and a proposed study population and researchers from a developing country. In each region, the case was translated from English in written or oral form into one or more languages, depending on the preference of the local organisers.

An introductory session outlined the important scientific aspects of HIV preventive vaccines, and the basic principles of biomedical research ethics. However, no ethical guidance documents or statements were referred to during the course of the workshops, unless they were introduced by the participants. A set of open-ended questions was used to facilitate discussion on specific topics (for example, informed consent), with the same questions being used in all three regions. The case was discussed in depth in small groups, and then further with all participants at the table. Important ethical issues were identified by the group, and on many of these issues a consensus was reached. For those issues on which consensus could not be reached, the points of controversy were defined and articulated. Final statements were written and agreed upon separately for each of the three workshops. An additional workshop was organised in 
Washington, DC, USA in May, 1998 to discuss specific issues related to bilateral collaboration.

The outcomes of the workshops were presented at a meeting in Geneva in June, 1998, along with a draft document that condensed these outcomes into a set of proposed ethical guidance statements. About 70 individuals participated in this discussion, including representatives from each of the regional workshops, the World Health Organisation, the Council of International Organisations of Medical Sciences (CIOMS), UNAIDS, research funding agencies in developed countries, public health organisations, activist groups, biomedical and social scientists, ethicists, and the media.

\section{Outcomes of the consultation process}

Each of the three regional workshops discussed the same general topics. The results of these discussions are summarised in the following sections.

'DEVELOPING COUNTRY' AND 'DEVELOPED COUNTRY' TERMINOLOGY

Participants from all of the regions expressed their concern about the inadequacy of terminology used to categorise countries of advantage and disadvantage. Previous ethical documents have drawn a distinction between "developed" and "developing" countries or communities. "Developing" countries have generally been perceived as deserving of additional protection to prevent harm or exploitation as a result of biomedical research. However, the usefulness of the developing/developed terminology for assessing risk of harm and exploitation was seen to be limited. It refers primarily to economic considerations, while many other factors are relevant in HIV vaccine research. It also separates nations into two categories, whereas in reality they are distributed along a spectrum.

In place of the developing/developed terminology, each region described the factors that are likely to influence the degree of vulnerability of the prospective subject population to exploitation or harm. These include the following:

1. Limited economic development;

2. Inadequate protection of human rights in general, and more specifically, discrimination on the basis of HIV antibody status;

3. Inadequate community/cultural experience with, or understanding of, scientific research;

4. Limited political awareness of the importance and process of vaccine research;

5. Limited availability of health care and treatment options;
6. Limited ability of individuals in the community to provide informed consent, often based on class, gender, etc;

7. Insufficient formal experience with, or capability to conduct, ethical or scientific review of proposed research, and

8. Insufficient infrastructure and technical capacity to conduct the proposed research.

Most countries and communities are characterised to varying degrees by some or all of these conditions. However, as the number or severity of these characteristics increases, the potential for harm or exploitation also increases. These factors contribute to a disparity in real or perceived power between sponsor(s) and host countries, make it difficult for residents of a host country to make informed, reasoned and independent choices, and increase the potential for social harm to occur.

URGENCY FOR VACCINE DEVELOPMENT VERSUS PROTECTION OF HUMAN SUBJECTS

One major source of ethical tension appeared to be relevant to many of the specific topics discussed during the consultation process. The experience of the HIV epidemic was clearly desperate for many of the developing countries participating in the workshops. The moral charge to expedite development of an effective vaccine appeared at times to conflict with the ethical imperative to safeguard the rights and welfare of communities and individuals participating in vaccine trials, especially when such safeguards would require time to put into place. Workshop participants struggled to resolve this tension. The degree to which resolution was reached is reflected in the sections that follow.

\section{SELF-DETERMINATION VERSUS PROTECTIONISM}

Many of the developing countries represented at the workshops endeavoured to define their own level of readiness to take responsibility for determining whether to participate in vaccine trials. Current ethical guidelines that address research in developing countries lean in the direction of protectionism. For example, the conduct of phase I and II trials in developing countries has been discouraged. ${ }^{2}$ The rationale is that there is a greater risk inherent with early trials, and that the potential for exploitation or harm in these settings cannot be justified. Participants from all the regions concluded, however, that, with certain safeguards in place, it would be appropriate for developing countries to participate in phase I and II clinical trials when there was a valid scientific rationale. This might expedite the progression to phase III efficacy trials, and may be the only way 
to determine safety and immunogenicity for the specific subtype of virus being targeted in the host country. In addition, phase I and II trials would provide host countries with important capacitybuilding experience in reviewing and conducting research, and building public and political support prior to the much larger and more complex phase III trials.

Conditions that would need to be fulfilled prior to conducting HIV vaccine trials in developing countries might include the following:

1. The vaccine is expected to be effective against a strain of HIV virus that is a relevant public health problem in the host country;

2. The host country has, or with assistance can develop, adequate scientific capability and administrative infrastructure for the successful conduct of the proposed research;

3. The host country has, or with assistance can develop, the capability to conduct scientific and ethical review, and

4. Community members, policy makers, ethicists and researchers in the host country have determined that their residents will be adequately protected from harm or exploitation, and that the vaccine development programme is responsive to the health needs and priorities in their country.

ACHIEVING RESEARCH OBJECTIVES VERSUS

PREVENTING HIV INFECTION

Efficacy trials of HIV vaccines are successful only if new infections are occurring among trial participants. It is necessary to demonstrate a difference in incidence between the control group and the vaccinated group if efficacy is to be proven. However, it is also true that HIV can be prevented with reductions in risk behaviour Although most risk behaviour modification strategies have proven insufficient to reduce HIV incidence to zero, the most rigorous approaches have been proven significantly to decrease the rate of new infections. This introduces what might be perceived as a conflict of interest between researchers, who hope to prove that the vaccine is effective, and trial participants, whose interest it is to defend themselves from infection by any methods possible.

Throughout the consultation process, contributors agreed that "high quality" HIV prevention counselling must be provided for all trial participants. Exactly what information should be conveyed through what specific methods was not agreed upon. The degree to which items such as condoms and sterile syringes should be made available remained a point of controversy, al- though some degree of access to these was broadly supported. In addition, some suggested that those responsible for providing prevention strategies should be independent, or at least at arm's length, from those whose main interest was the research.

TREATMENT AND CARE FOR PARTICIPANTS CONTRACTING HIV

The most contentious issue arising from the consultation process was whether there is an ethical imperative for participants in an HIV vaccine trial to be provided with treatment, should they become infected during the course of the trial. Infection would likely occur as a result of ongoing risk behaviour in some who are assigned to a control arm, and also in some assigned to the arm receiving the candidate vaccine if it failed to be protective. Workshop participants within each region reached a consensus on the question of treatment, but the consensus reached was different for each region. Positions ranged from providing the "best proven" treatment, to providing the level of treatment that is readily available within the host country. This debate has continued to the present. Several specific questions have arisen. Is there an ethical obligation for trial sponsors and investigators to provide treatment when the intervention being tested is a vaccine rather than a therapy? Is the cost of providing treatment likely to be prohibitive for conducting vaccine trials in developing countries? Is providing treatment likely to constitute an unreasonable inducement to participate in a trial when there is minimal treatment available to the general population? Once treatment is started, is there an ethical obligation to provide this beyond the duration of the trial, and, if there is an ethical justification for providing treatment, what components should be included in the treatment package?

The justification for providing treatment has been made from several perspectives. It might be hypothesised (although it has not been proven) that participation in an HIV preventive vaccine trial could lead to increased risk behaviour among the participants. Those who participate in vaccine trials will be informed that they should not consider themselves to be protected from infection, since neither the placebo nor the candidate vaccine are known to be effective. They will also be informed of the behaviours that will put them at risk of infection, and how to avoid exposure to HIV. However, it is possible that even with this information, research subjects will believe that they are protected to some degree by their participation in the trial, and will increase their risk behaviour, making HIV infection more likely. Under this hypothesis, it is not the vaccine itself 
that causes an increase in HIV risk, but rather people's beliefs and perceptions about the significance of receiving an experimental vaccine.

\section{Global social justice}

Another perspective arises from the framework of global social justice. This argument points to the disparity in economic resources that exists between countries. HIV preventive vaccine trials will likely be funded by sponsors from countries with greater wealth and better health care than the host country populations in which they are eventually tested. In many of the potential host countries, there is no treatment available to the general population. In addition, sponsor companies are likely to profit from the eventual sale of preventive HIV vaccines. Providing treatment to those infected with HIV during vaccine trials in developing countries would be a step towards addressing an ethical obligation for international researchers to contribute towards equality of resources.

Those who agree on providing treatment must eventually identify the components that would be included in an ethical treatment package. The focus on combination antiretroviral medication is a relatively recent phenomenon. Although this is clearly the most effective therapy that has been available since the epidemic was recognised, it continues to be extremely expensive, noncurative, complex to administer and monitor, and accompanied by numerous adverse effects. Treatment and prevention of opportunistic infections, palliative care, nutrition, spiritual support, psychological support and rehabilitation, among others, are also important components of care for those infected with HIV.

Some have proposed that appropriate treatment is "the best proven therapy" anywhere in the world. This reference to the Declaration of Helsinki implies that all proven treatment modalities relevant to HIV and its complications should be made available for the lifetime of the individual. ${ }^{5}$ There are several barriers to providing "best proven therapy". First, it is often difficult for experts to reach consensus on what the best proven treatment is. Then, if consensus is reached, economic cost and technical feasibility play a role in locations where infrastructure in services such as health care, laboratory and transportation are not secured. Without adequate infrastructure for monitoring individuals taking complex medical regimens, there could be a significant risk of harmful effects as a result of treatment. Finally, if expensive and sophisticated treatment is provided in a context where no treat- ment is generally available, this may constitute undue inducement to participate in a trial.

An alternative proposal arising from the consultations was to provide access to a level of treatment that approaches as closely as possible the best proven treatment, that is, "the highest attainable". Sponsors could consider ways in which their activities might build up not only research capacity in a host country, but also capacity to deliver health services. Efforts could be made to build this capacity in ways that are likely to be integrated into the infrastructure of the host country, and are not likely to disappear when the research is completed. As an example, this could happen through the reinforcement of laboratories, health centres and non-government organisations in the host country.

During the process of consultation there was general agreement that where no treatment is available to the general population, sponsors and investigators are obligated to ensure that some form of treatment is made available to study participants. However, whether an international ethical standard should uphold the provision of the best proven treatment, the highest attainable treatment, or a procedure for decision making where controversy persists, is still a point of vigorous discussion in the global community.

ACCESS AND AVAILABILITY OF A PROVEN HIV VACCINE Participants in all regions expressed disapproval of the historic practice of testing pharmaceutical products in developing countries without ensuring access for residents of the host country to successful products resulting from the research. Agreement was not reached on how accessibility could be ensured, nor on how broadly a new product should be made available. Workshop participants acknowledged the complexity of designing economic and political mechanisms capable of ensuring availability even before a product has been tested. There was consensus that volunteers who receive a placebo in a vaccine trial should be offered the final vaccine product once it is approved and licensed. Others who were considered to be candidates for such a vaccine were those at risk of infection in the general population of the host country, and similar populations in other developing countries. However, whether access and availability should be extended to this degree remains a point of debate.

Effective incentives for fostering development of HIV vaccines, and for ensuring the ultimate availability of vaccines to the populations that need them most, were identified as an area of priority for future work. Suggested strategies included financial rewards through the market place 
and public subsidies, technology transfer, and negotiation of intellectual property.

\section{Comment}

This process of international consultation has resulted in several notable outcomes. Most importantly, individuals in countries that are relatively unfamiliar with biomedical research and research ethics have become part of an ongoing, informal discussion on the ethical dilemmas encountered in HIV vaccine trials. This global discussion is critical. It constitutes one step toward equalising what is likely to be an inherently unequal balance of power between collaborating countries. Through enhanced capacity to participate in ethical debate - to analyse and articulate the ethical issues most relevant in the local context - countries and communities anticipating involvement in research will be better equipped to ensure that the rights and welfare of human subjects are safeguarded. To this end, UNAIDS has proceeded to initiate a series of training workshops on ethics for members of ethical review committees and community representatives in developing countries.

It was anticipated that the consultation process would lead to the development of an ethical guidance document that could provide direction to those involved in planning and reviewing international HIV vaccine research. Since the final meeting in Geneva in June, 1998, a draft document has been prepared and distributed for comment. It was clear from the June, 1998 meeting and from the responses to the draft that a consensus had been reached on a substantive ethical standard for many of the identified issues. However, consensus could not be reached on a substantive solution to the question of treatment for those infected with HIV during the course of a trial. Although some have suggested that international guidelines ought to uphold a standard for a decision making procedure for such areas of ethical controversy, many have expressed concern with an approach that does not offer a substantive standard to be applied globally. UNAIDS plans to publish its own position statement and guidance in the near future.

Because these consultations were carried out in regions of the world far removed from one another culturally as well as geographically, it might be thought that lack of agreement on some issues stemmed from cultural differences. There is no denying that different cultures and subgroups within societies exhibit a variety of moral beliefs and practices. These empirical facts yield the descriptive thesis known as "cultural relativity". It is certainly true that southeast Asian nations, countries in South America and the Caribbean, and the region of east Africa have many cultural variations in beliefs and practices. But as striking as these variations may be, they did not play a role in those instances where participants in the consultations reached different conclusions about what was ethically required. Nor was cultural relativity a factor in failure to arrive at consensus in the final meeting in Geneva, at which several additional countries and regions were represented.

Where one might have expected some disagreement stemming from cultural diversity, for example, in the requirements for individual informed consent to participate in vaccine trials, there was striking uniformity of agreement among the three regional consultations. Although procedural steps in obtaining consent may vary from one country or region to another, participants in all three regional consultations unanimously concurred that in no circumstances, such as cultures in which women are normally not accorded decisionmaking authority, may the requirement for individuals to provide voluntary, informed consent on their own behalf be abandoned or weakened.

\section{Economic disparity}

The lack of consensus that did emerge on several points had little to do with different norms or values in different countries and everything to do with the economic disparity between industrialised and resource-poor countries, and with countries along the spectrum of economic development. Resulting from these economic disparities is a different level of medical care in wealthier countries and poorer nations. The chief point of controversy, as noted above, was whether there is an ethical obligation to provide treatment for HIV and its associated complications for participants in an HIV vaccine trial. Disagreements about whether such an obligation exists, and if so, who bears that responsibility, arise from different views about what justice requires and what wealthy sponsors owe to resource-poor countries in which the research is carried out.

A remaining unanswered question is whether an ethical double standard exists if participants in vaccine trials who become infected in some countries are assured access to the best proven therapy, while those in other countries will never be provided with treatment. One side in this debate points to the provision in the Declaration of Helsinki that requires assurance of access to the best proven therapy, ${ }^{5}$ and argues that to depart from that principle is to adopt a double standard. This side contends that ethical principles may not be 
"relativised" to different economic circumstances. In contrast, as one commentator observed regarding an analogous controversy: "The real double standard lies not in the way the trials are being conducted, but in the inequity in access to medicines in different countries". ${ }^{6}$ On the latter view, ethical obligations may be relativised to different economic circumstances and disparities in the health infrastructure in different countries. However that debate is to be resolved, it is not a case of ethical relativism stemming from cultural variation.

\section{Disclaimers}

This article does not represent the official position of UNAIDS. A guidance document on ethical considerations in international HIV vaccine trials is to be released which will describe the UNAIDS policy.

\section{Acknowledgements}

We would like to thank Claire Pattou for organising the regional workshops, Bob Levine for sharing with us his substantial experience in this area and for preparing initial drafts of the guidance document, and Barry Bloom for his continuing support, direction and inspiration. We also wish to thank the Joint United Nations Programme on HIV/AIDS which funded this project.
Dale Guenter, MD, is Assistant Professor in the Department of Family Medicine, McMaster University, Hamilton, Canada. Fose Esparza, MD, is Leader, Vaccine Team, the foint United Nations Programme on HIVIAIDS (UNAIDS), Geneva, Switzerland and Ruth Macklin, PhD, is Professor of Bioethics in the Department of Epidemiology and Social Medicine, Albert Einstein College of Medicine, New York, USA and Chair, UNAIDS Ethical Review Committee, Geneva, Switzerland.

\section{References}

1 World Medical Association. Declaration of Helsinki. Somerset West, Republic of South Africa: $48^{\text {th }}$ General Assembly, World Medical Association, 1996.

2 Council for International Organisations of Medical Sciences. International ethical guidelines for biomedical research involving human subjects. Geneva: Council for International Organisations of Medical Sciences (CIOMS) in collaboration with the World Health Organisation (WHO), 1993.

3 International Conference on Harmonisation of Technical Requirements for Registration of Pharmaceuticals for Human Use (European Union, Japan, USA).Guideline for good clinical practice (ICH harmonised tripartite guideline), 1997.

4 World Health Organisation. Guidelines for good clinical practice (GCP) for trials on pharmaceutical products. Geneva: WHO technical report series, no $850,1995$.

5 See reference 1:article II. 3.

6 Piot P. Ethics of placebo-controlled trials of zidovudine to prevent the perinatal transmission of HIV in the Third World. [letter]. New England fournal of Medicine 1998;338:839. 